\title{
Use of FLAIR Imaging to Identify Onset Time of Cerebral Ischemia in a Canine Model
}

\author{
X.-Q. Xu, Q.-Q. Zu, S.-S. Lu, Q.-G. Cheng, J. Yu, Y. Sheng, H.-B. Shi, and S. Liu
}

\begin{abstract}
BACKGROUND AND PURPOSE: Stroke is a leading cause of death and disability, and many studies have focused on the evolution of FLAIR imaging in the acute and chronic time window. The purpose of this study was to evaluate the potential efficacy of FLAIR-related techniques in identifying the onset time of cerebral ischemia in a canine embolic stroke model.
\end{abstract}

MATERIALS AND METHODS: An embolic ischemic model was generated through the use of an autologous clot in 20 beagle dogs. Both FLAIR and DWI were performed at 3 hours, 4 hours, 5 hours, 6 hours, and 24 hours after embolization, respectively. Visual "DWI-FLAIR mismatch" was defined as hyperintense signal detected on DWI but not on FLAIR. The relative signal intensity of FLAIR-positive lesions and the degree of DWI-FLAIR mismatch was calculated as relative FLAIR = relative signal intensity of FLAIR positive lesions, mismatch degree $=$ $\left(100-\mathrm{V}_{\text {FLAIR }} / \mathrm{V}_{\mathrm{DWI}}\right) \times 100 \%$.

RESULTS: The ischemic model was successfully established in all animals. FLAIR-positive lesions were seen in 3, 11, 16, 19, and 20 beagle dogs at 5 time points after embolization, respectively. There was significant correlation between the relative FLAIR, degree of DWI-FLAIR mismatch, and the onset time (relative FLAIR: $r=+0.42 ; 95 \% \mathrm{Cl}, 0.20-0.60$; mismatch degree: $r=-0.85 ; 95 \% \mathrm{Cl}, 0.89-0.78$ ). Receiver operating characteristic curves showed that the degree of DWI-FLAIR mismatch could identify the hyperacute ischemic lesions with a sensitivity range from 1.00-0.76; visual DWI-FLAIR mismatch sensitivity ranged from $0.85-0.39$, whereas specificity was $0.83-0.95$ versus $0.85-1.00$.

CONCLUSIONS: The relative FLAIR and DWI-FLAIR mismatch values were useful in predicting the onset time in our canine embolic stroke model. The degree of DWI-FLAIR mismatch proposed in our study could be a good indicator with high sensitivity for identifying the hyperacute ischemic stroke.

ABBREVIATIONS: $r S I$ = relative signal intensity; DWI-FLAIR mismatch = DWI positive and FLAIR negative; ROC = receiver operating characteristic; $r$ FLAIR $=$ relative signal intensity of FLAIR positive lesions; $\mathrm{SI}=$ signal intensity; $r A D C=$ relative $A D C$

ntravenous administration of $\mathrm{tPA}$ is the only proven, effective treatment for acute ischemic stroke within the first 4.5 hours after onset of symptoms. ${ }^{1,2}$ However, an estimated $25 \%$ of ischemic strokes occur during sleep, and the exact time of the onset of symptoms is unclear, which means that a large group of patients is precluded from the time-based thrombolytic therapy, especially

Received April 20, 2013; accepted after revision May 23.

From the Department of Radiology, First Affiliated Hospital of Nanjing Medical University, Nanjing, China.

This research was supported by National Natural Science Foundation of China (81000653 to S. Liu; 30870710 to H.B. Shi)

Xiao-quan Xu and Qing-quan Zu contributed equally to this work.

Please address correspondence to Sheng Liu, MD, PhD, Department of Radiology, First Affiliated Hospital of Nanjing Medical University, 300 Guangzhou Rd, Nanjing 210029, China; e-mail: liusheng@njmu.edu.cn

- Indicates open access to non-subscribers at www.ajnr.org

http://dx.doi.org/10.3174/ajnr.A3689 in developing countries. ${ }^{3}$ Therefore, a new diagnostic method that can identify the onset time of stroke is urgently required.

Recently, FLAIR sequences of MR imaging in acute stroke have attracted more attention as a potential surrogate marker for time since stroke onset. ${ }^{4,5}$ Specifically, a visual mismatch between DWI and FLAIR images has been shown to identify patients likely to be within a time window of 4.5 hours. ${ }^{6-8}$ Although these findings showed the high specificity of a "DWI-FLAIR mismatch" as a "tissue clock" to identify patients potentially eligible for thrombolytic therapy, some limitations of the method remained, such as different interobserver agreement rate, patient selection bias, and especially the relatively low sensitivity.

To overcome these limitations, we performed several quantitative measurements with the use of FLAIR images, including relative signal intensity (rSI) of FLAIR lesions, degree of DWI-FLAIR mismatch, and also visual DWI-FLAIR mismatch, to evaluate the potential efficacy of FLAIR-related techniques and to identify the onset time of acute cerebral ischemia in a beagle dog model. ${ }^{9,10}$ 


\section{MATERIAL AND METHODS}

\section{Animal Preparation and Model Establishment}

The surgical procedures and experimental protocol were approved by the Institutional Animal Care and Use Committee of Nanjing Medical University (Nanjing, China). Effective actions were taken for reducing pain or discomfort during the experiments.

A total of 20 adult healthy beagle dogs of either sex, weighing $12-15 \mathrm{~kg}$, were anesthetized with intravenous injection of $3 \mathrm{mg} / \mathrm{kg}$ of pentobarbital (Pentobarbital Sodium Salt, Chemical Reagent Company, Shanghai, China). The airways were secured by means of oral endotracheal tubes with spontaneous respiration. Bilateral femoral arterial and left femoral venous accesses were obtained by use of $5 \mathrm{~F}$ sheaths for catheterization, physiologic monitoring, and drug administration. Body temperature was maintained at 37$39^{\circ} \mathrm{C}$ during the interventional procedures and the duration of recovery after procedures by use of heating blankets. Sterile procedures were strictly used in all cases.

The beagle dog's cerebral ischemic models were established through the use of a method similar to the one used in our previous reports. ${ }^{9,10}$ Briefly, a prepared autologous clot (approximately $1.7 \mathrm{~mm}$ in diameter and $5 \mathrm{~mm}$ in length) was injected into the left proximal MCA under live fluoroscopy, and embolization was confirmed by angiography. After that, a 5F catheter was guided to $2 \mathrm{~cm}$ distal to the orifice of the ipsilateral ICA to block the blood flow for 2 hours. The animals were then transported to the MR imaging suite for imaging studies.

\section{MR Imaging}

Imaging was performed with a 3T MR system (Magnetom Trio; Siemens, Erlangen, Germany), with the use of a transmit-receive extremity coil with a diameter of $15 \mathrm{~cm}$. Imaging acquisitions were performed serially at 3 hours, 4 hours, 5 hours, 6 hours, and 24 hours after the left MCA embolization, respectively. The same medications and doses were used to maintain immobility and sleep during the MR imaging scan. We checked T2-weighted images (acquisition matrix $=320 \times 320, \mathrm{TR}=5000 \mathrm{~ms}$, $\mathrm{TE}=76$ $\mathrm{ms}$ ), FLAIR (acquisition matrix $=320 \times 320$, TR $=8000 \mathrm{~ms}$, $\mathrm{TE}=97 \mathrm{~ms}$ ), and DWI (acquisition matrix $=320 \times 320, \mathrm{TR}=$ $5500 \mathrm{~ms}, \mathrm{TE}=97.3 \mathrm{~ms}$ ). After MR examination, all animals recovered and were kept in the animal facility for other studies.

\section{Imaging Assessment}

FLAIR-positive (FLAIR+) or DWI-positive (DWI+) was defined as new hyperintense signals detected on FLAIR or DWI. For signal intensity (SI) changes of ischemic lesions on FLAIR, an ROI was defined on 1-3 sections showing the most obvious lesion completely covering the ischemic area. Control SI values were obtained from an ROI drawn contralaterally. Two independent raters (Y. Sheng, Q.G. Cheng) judged FLAIR+ according to acute lesions on DWI. Relative SI was obtained from FLAIR images according to the following formula: $\mathrm{rSI}=$ lesion SI/contralateral SI.

For ADC changes of ischemic lesion on DWI, an ROI was also defined on the sections showing the most obvious lesion. Control ADC values were obtained from an ROI drawn contralaterally. The relative ADC ( $\mathrm{rADC}$ ) value was obtained according to the following formula: $\mathrm{rADC}=$ lesion ADC/contralateral ADC.
"DWI-FLAIR mismatch" was defined as new hyperintense signal detected on DWI but not on FLAIR. The sensitivity and specificity values were calculated for the allocation of dogs to time interval from symptom onset to MR imaging scan within 3 hours, 4 hours, 5 hours, and 6 hours by DWI-FLAIR mismatch.

The volume of DWI+ and FLAIR + lesions at each time point were also calculated. For the quantification of lesion size on DWI and FLAIR, the lesions in the same section were delineated by 3 authors (X.-Q. Xu, S.-S. Lu, Q.-Q. Zu), with consensus by use of an operator-defined ROI on each of the lesion-containing sections. The lesion volumes were obtained by multiplying the lesion areas by the section and gap thickness. Through the use of the volume of DWI+ and FLAIR + lesions, the degree of DWI-FLAIR mismatch was calculated according to the following formula: Degree $=\left(100-\mathrm{V}_{\mathrm{FLAIR}} / \mathrm{V}_{\mathrm{DWI}}\right) \times 100 \%$. During the whole imaging assessment process, if any discrepant results occurred between 2 evaluators, the third and senior evaluator (S. Liu) would make the final decision.

\section{Statistical Analysis}

Interobserver and intraobserver agreement for the rating of FLAIR, DWI, and ADC images was assessed by means of Pearson correlation coefficient. The correlation between rFLAIR and onset time of stroke, and the correlation between the degree of DWIFLAIR mismatch and the onset time were assessed by Spearman $\rho$ analysis. The linear regression curve was plotted by use of GraphPad Prism statistical analysis software (GraphPad Software, San Diego, California). An optimal cutoff value was determined from the receiver operating characteristic (ROC) curve to analyze if there were critical ADC values that were sensitive or specific for FLAIR positivity. An ROC curve was used to identify the optimal cutoff value to allocate the ischemic lesions within 3 hours, 4 hours, 5 hours, and 6 hours with degree of DWI-FLAIR mismatch. The numeric data were averaged over all animals and reported as mean \pm standard deviation. A significant difference was considered if the $P$ value was $<.05$. Statistical analysis was carried out with SPSS 17.0 (IBM, Armonk, New York).

\section{RESULTS}

\section{Ischemic Model}

All 20 cerebral ischemic models were established successfully without any procedure-related complications or casualties (Fig 1). DWI+ lesions were seen in all 20 beagle dogs starting from 3 hours after embolization. The DWI indicated that the cerebral ischemic lesions were located on the ipsilateral caudate nucleus and the cortical area of the temporal lobe. Generally, the ischemic lesions were first found at the caudate nucleus, followed by the lesions located in the cortical area of the temporal lobe (Fig 2).

\section{Correlations Between rFLAIR with Time after the Model Was Established}

Intraobserver agreement for qualitative judgment of FLAIR lesion visibility of observer 1 and observer 2 was $87 \%(k=0.78$; 95\% CI, $0.77-0.98)$ and $85 \%(k=0.77 ; 95 \%$ CI, 0.75-0.96), respectively. Intraobserver agreement for quantitative judgment of FLAIR+ lesions of observer 1 and observer 2 was $89 \%(k=0.81 ; 95 \% \mathrm{CI}$, $0.80-0.99)$ and $91 \%(k=0.84 ; 95 \%$ CI, 0.82-0.98), respectively. 

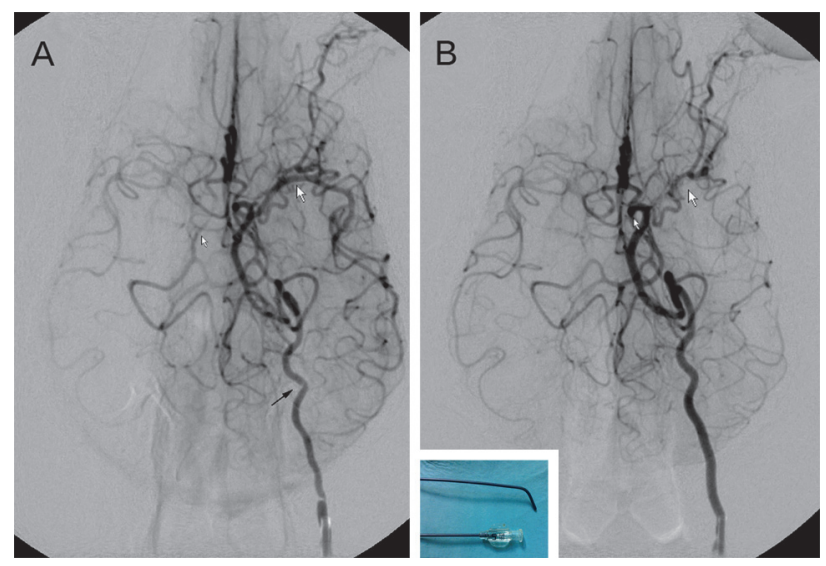

FIG 1. Representative real-time DSA images before and after MCA embolization in beagle dogs. A, Angiography clearly demonstrated the anatomy of intracranial arteries, including the left MCA trunk (large arrowhead), tortuous ICA (black arrowhead), and cerebral circulation (small arrowhead) through the left ICA injection before embolization. $B$, At the arterial phase, angiography immediately after thrombus injection through the catheter, the main trunk of MCA was completely occluded (small and large arrowheads). After that, a $5 \mathrm{~F}$ catheter was guided to $2 \mathrm{~cm}$ distal to the orifice of ipsilateral ICA to block the blood flow for 2 hours. The catheter used in our study is displayed in left-bottom inset.

Interobserver agreement for qualitative judgment of FLAIR lesion visibility was $80.1 \%(k=0.52$; $95 \%$ CI, $0.56-0.91)$. Interobserver agreement for quantitative judgment of rFLAIR lesion was $86 \%$ ( $k=0.77$; 95\% CI, 0.76-0.98).

FLAIR + lesions were seen in 3 of the $20(15 \%)$ beagles at 3 hours after embolization, 11 of the $20(55 \%)$ beagles at 4 hours after embolization, 16 of the $20(80 \%)$ beagles at 5 hours after embolization, 19 of the 20 (95\%) beagles at 6 hours after embolization, and all $20(100 \%)$ beagles at 24 hours after embolization, respectively (Fig 3 ).

All 69 FLAIR + lesions had a mean rSI of $1.40 \pm 0.27$. The rFLAIR values were $1.40 \pm 0.04,1.23 \pm 0.13,1.27 \pm 0.03,1.59 \pm$ 0.09 , and $1.43 \pm 0.07$ at 3 hours, 4 hours, 5 hours, 6 hours, and 24 hours after embolization, respectively. In a linear regression model, there were significant correlations between rFLAIR lesions and onset time $(P<.05)$. The Spearman correlation coefficient for rFLAIR and onset time was +0.42 (95\% CI, 0.20-0.60) (Fig 4).

\section{Correlation Between FLAIR+ Images and the rADC Threshold Value}

The ROC curve analysis results indicated that the rADC value of 0.54 might be the critical threshold value. With the rADC value of 0.54 set as the diagnostic threshold value, the best sensitivity and specificity for judging FLAIR positivity were 0.52 and 0.68 , respectively (Fig 5).

\section{Application of DWI-FLAIR Mismatch in the Ischemic Model}

According to the pattern of DWI-FLAIR mismatch, the interval time between onset and image acquisition was presumed to be within 3 hours, sensitivity of 0.85 and specificity of 0.85 ; within 4 hours, sensitivity of 0.65 and specificity of 0.92 ; within 5 hours, sensitivity of 0.50 and specificity of 0.97 ; and within 6 hours, sensitivity of 0.39 and specificity of 1.00 .
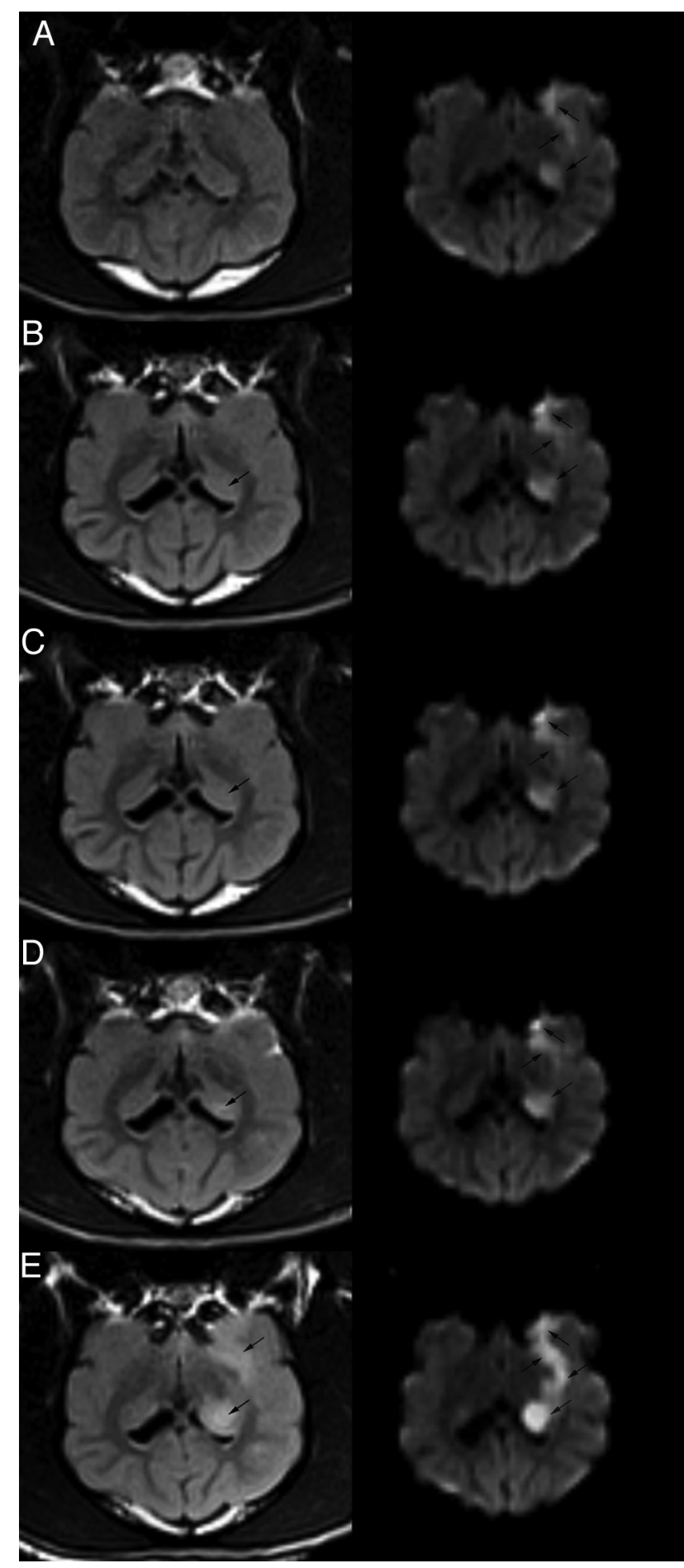

FIG 2. Sequential DWI and FLAIR images of coronal sections. The DWI and FLAIR were performed at 3 hours (A), 4 hours (B), 5 hours (C), 6 hours (D), and 24 hours ( $E$ ) after embolization, respectively. There was no signal abnormality at 3 hours on the FLAIR image (A, left). Relative slightly high signal intensity area (arrow) with ovoid shape was observed in the left caudate nucleus on 4-hour FLAIR image ( $B$, left). Increased high signal intensity was seen at 5 hours, 6 hours, and 24 hours on the FLAIR image ( $C-E$; left), and lesions in the cortical area were seen on the 24-hour FLAIR image (E, left). Sequential DWI of coronal sections was all positive from 3 hours to 24 hours after model establishment (A-E; right).

Meanwhile, the degree of DWI-FLAIR mismatch was also calculated by means of the formula: degree $=\left(100-\mathrm{V}_{\text {FLAIR }} /\right.$ $\left.\mathrm{V}_{\text {DWI }}\right) \times 100 \%$. Interobserver agreement for quantitative judg-

AJNR Am J Neuroradiol 35:311-16 Feb 2014 www.ajnr.org 313 


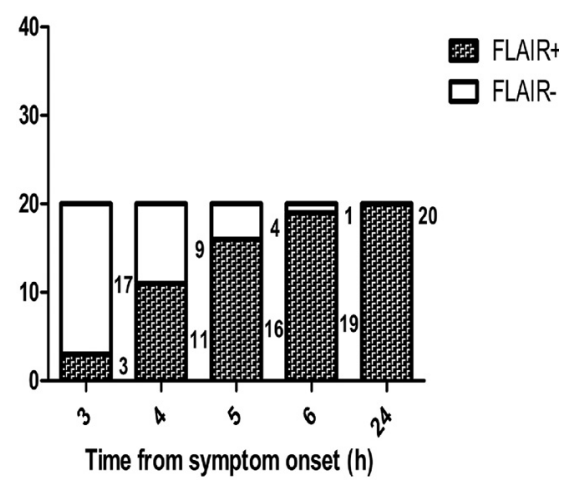

FIG 3. Proportion of FLAIR-negative and FLAIR-positive results on the basis of the 5 onset time points in the 20 beagle dogs. Number of FLAIR-negative and FLAIR-positive dogs are presented in bars.

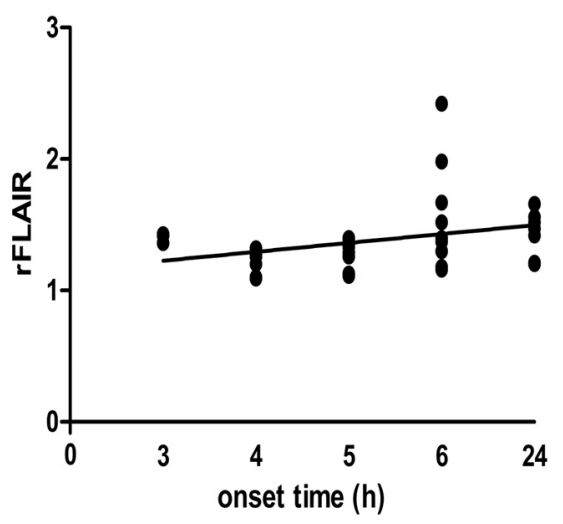

FIG 4. Relative SI on FLAIR images and time interval from onset to MR imaging scanning. Significant correlation between the rFLAIR and the onset time was found $(r=+0.42 ; 95 \% \mathrm{Cl}, 0.20-0.90)$.

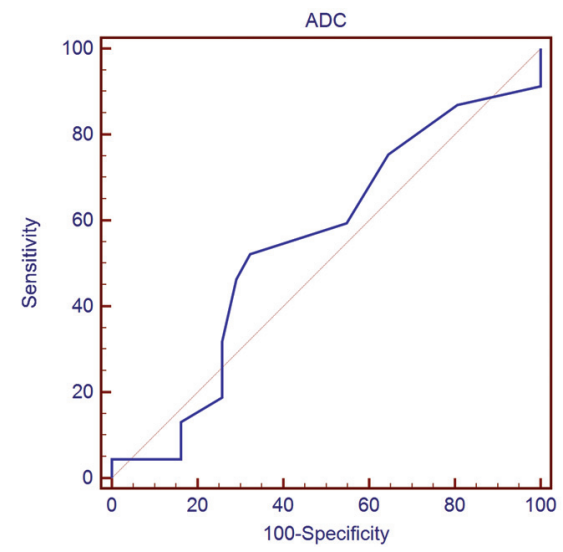

FIG 5. ROC of the rADC value in identifying the FLAIR + lesions. ROC curve showed that the rADC value of 0.54 might be the critical threshold value that could quantify FLAIR + lesions with the optimal sensitivity and specificity ( 0.52 and 0.68 , respectively).

ment of "degree of mismatch" was 79\% ( $k=0.77$; 95\% CI, $0.72-$ 0.84). The degree of DWI-FLAIR mismatch was $0.99 \pm 0.00$, $0.93 \pm 0.01,0.81 \pm 0.11,0.67 \pm 0.06$, and $0.42 \pm 0.10$ at 3 hours, 4 hours, 5 hours, 6 hours, and 24 hours after embolization, respectively. In a linear regression model, there is a significant correlation between degree of DWI-FLAIR mismatch and onset time $(P<.05)$. The Spearman correlation coefficient for degree of

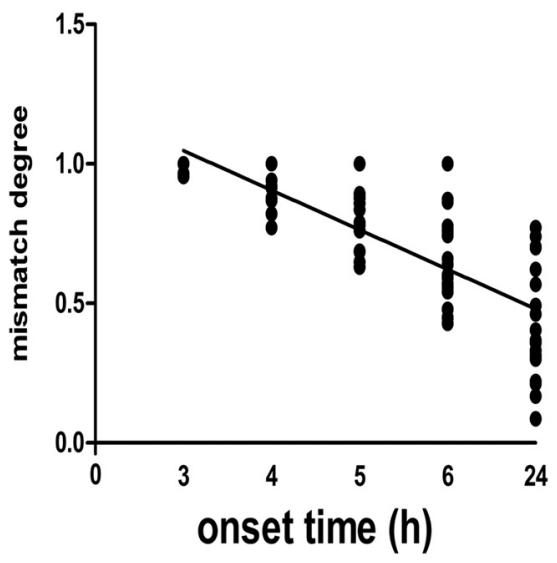

FIG 6. Degree of DWI-FLAIR mismatch and time interval from onset to MR imaging scanning. Significant correlation between the degree of DWI-FLAIR mismatch and the onset time was found $(r=-0.85$; $95 \% \mathrm{Cl}, 0.89-0.78)$.

DWI-FLAIR mismatch and onset time was - 0.85 (95\% CI, $0.89-$ 0.78) (Fig 6).

The ROC analysis results indicated that the mismatch degree value of 0.95 might be the critical threshold value to identify the ischemic lesion within 3 hours, with a sensitivity and specificity of $100 \%$ and $82.5 \%$, respectively. The mismatch degree value of 0.90 might be the critical threshold value to identify the ischemic lesion within 4 hours, with a sensitivity and specificity of $85 \%$ and $91.7 \%$, respectively. The mismatch degree value of 0.79 might be the critical threshold value to identify the ischemic lesion within 5 hours, with a sensitivity and specificity of $83.3 \%$ and $90 \%$, respectively. The mismatch degree value of 0.75 might be the critical threshold value to identify the ischemic lesion within 6 hours, with a sensitivity and specificity of $76.2 \%$ and $95 \%$, respectively (Fig 7).

\section{DISCUSSION}

Our study demonstrates several major findings. First, the sensitivity of FLAIR for detecting hyperacute ischemic lesions clearly increases over time after embolization. Second, there is a significant correlation between the rFLAIR and the interval time from ischemic onset to MR imaging scanning. Thus, the rSI may be useful to predict the onset time of ischemic stroke. Third, also the most important, the new parameter, the degree of DWI-FLAIR mismatch proposed in our study, could be a better indicator for identifying the hyperacute ischemic stroke than visual DWIFLAIR mismatch. To our knowledge, this is the first study to test the potential efficacy of a FLAIR-related technique, especially DWI-FLAIR mismatch and degree of DWI-FLAIR mismatch in identifying the onset time of cerebral ischemia on the basis of an ischemic stroke animal model with known onset time. Our study design can effectively avoid the selection bias as reported by previous retrospective clinical studies.

Whether rFLAIR can serve as an indicator to predict the onset time of cerebral ischemia is still debated. Ebinger et $\mathrm{al}^{11}$ insisted that there was no significant correlation between rFLAIR and the onset interval time $(r=-0.15, P=.128)$, after analyzing 102 FLAIR + and 203 DWI+ lesions of 94 consecutive patients. However, Cheng et $\mathrm{al}^{12}$ declared that there was a moderately significant correlation between 

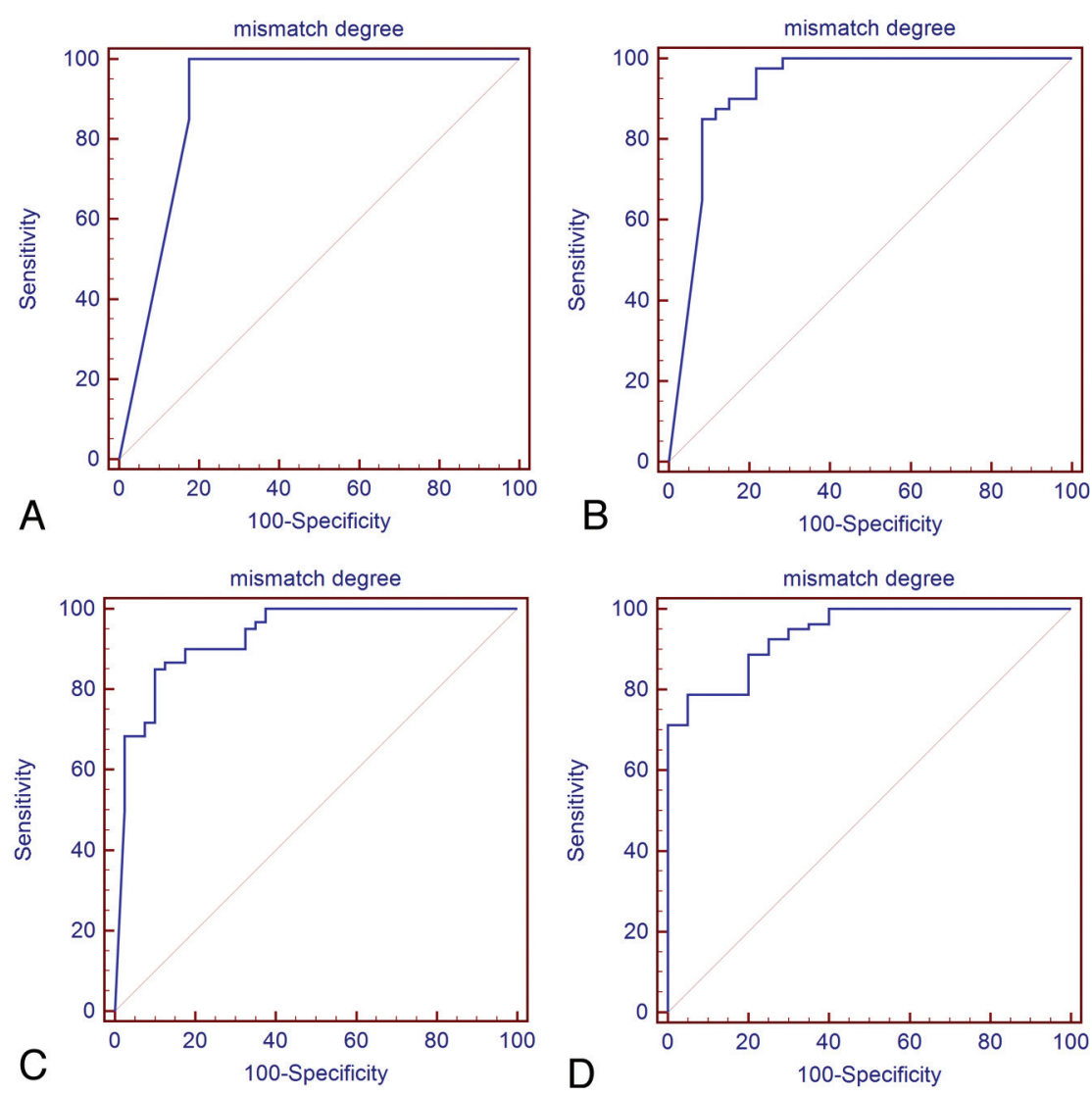

FIG 7. ROC of the DWI-FLAIR mismatch in identifying the hyperacute ischemic lesions within different onset time points. ROC showed that the cutoff values of DWI-FLAIR mismatch degree were $0.95,0.90,0.79$, and 0.75 , respectively, giving sensitivity of $1.00,0.85,0.83$, and 0.76 for identifying the hyperacute ischemic lesion at 3 hours, 4 hours, 5 hours, and 6 hours after embolization, respectively.

the rFLAIR and onset interval time $(r=0.38, P<.001)$, which is similar to opinions of Petkova et $\mathrm{al}^{13}(r=0.63, P<.001)$. Interestingly, the correlation ratio in our study was located between that of the latter 2 studies. Discrepancies in the results of correlations may partly be explained by patient characteristics. The median volume of FLAIR + lesions in the Cheng et al study was larger than that of the Ebinger et al study (4.5-10.5 mL versus $0.86-1.65 \mathrm{~mL})$. As a matter of course, the NIHSS score in the Cheng et al study was higher than that of the Ebinger et al study (7.3-8.8 versus 3-4). The larger ischemic volume and higher NIHSS score sometimes mean longer intervals in time from onset to MR imaging; therefore, more patients would be in the later time interval. Furthermore the statistical results would be different consequentially. However, in the Cheng et al study, data analysis was performed on images from multiple centers. This condition might lead to a heterogeneous set of FLAIR sequence parameters, which might contribute to a decreased homogeneity of rFLAIR, thus explaining a lesser correlation than that reported by Petkova et $\mathrm{al}^{13}$ and our study. Altogether, we think that a perspective, multiple center study with the uniform sequence parameters was needed to verify whether rSI of the FLAIR + lesions can serve as an alternative indicator to predict the onset time of cerebral ischemia.

Recently, a visual mismatch between DWI and FLAIR in acute stroke has attracted increased attention. Previous studies indicated that DWI-FLAIR mismatch could serve as a surrogate marker of lesion age. ${ }^{6-8}$ In our study, by use of this mismatch pattern to identify the hyperacute lesions within 3 hours, 4 hours, 5 hours, and 6 hours, we recorded specificities of $0.85,0.92,0.97$, and 1.00, respectively, which were similar to those in previous studies. As we know, onset time of acute ischemic stroke is critical for guidance of thrombolysis therapy. ${ }^{1,14,15}$ Earlier management sometimes means better prognosis and fewer complications. ${ }^{16}$ However, if patients whose onset time is beyond the time window receive thrombolytics therapy, the risk of intracranial hemorrhage would increase and the thrombolysis therapy would be harmful or even lethal. Because of this, high specificity of the pattern of DWI-FLAIR mismatch appears to be crucial in improving the safety of the thrombolytic therapy, especially for patients with unknown symptom onset. However, also similar to the previous study, the sensitivity of the use of DWI-FLAIR mismatch to identify the hyperacute lesions is relatively low $(0.39-0.85)$. So, if we choose the visual DWI-FLAIR mismatch as the criteria for identifying the acute stroke patients, only $39 \%$ of the patients (within 6 hours) would meet the criteria. As a result, more than half (61\%) of the patients would lose the chance of thrombolytic therapy.

Considering that visual DWI and FLAIR mismatch is a binary concept-and the varied interobserver agreement rate among previous studies - our study proposes the utility of a new quantitative parameter, degree of DWI-FLAIR mismatch, for determining the onset time of stroke. With the use of the new index, we found that there were significant correlations between degree of DWI-FLAIR mismatch and onset time $(r=-0.85$; 95\% CI, $0.78-$ 0.89). Meanwhile, by use of the optimal mismatch degree threshold identified by ROC analysis to identify the hyperacute ischemic lesions, we acquired markedly enhanced sensitivity compared with the initial visual mismatch analysis. At the same time, an increase of sensitivity did not result in an obvious decrease in specificity. Why the new mismatch index could effectively enhance the sensitivity is, we think, because the visual DWI-FLAIR mismatch represents the concept of "all or nothing." According to the criteria of visual DWI-FLAIR mismatch, there are just 2 results ("mismatch" or "no mismatch"), regardless of the ischemic lesions volume. Therefore, in our study, we transformed the binary DWI-FLAIR concept to a quantitative mismatch degree. The new mismatch degree index could effectively narrow the differences between each individual and each time point, rectify the influence of the lesion volume, and thus capture the difference that was omitted by the simple "yes or no" concept.

DWI and FLAIR techniques are more effective in identifying hyperacute strokes. First, we spent only approximately 45 
seconds and 3 minutes, 8 seconds, on DWI and FLAIR techniques, respectively. In general, approximately 4 minutes was needed to get the DWI and FLAIR image information. Considering the time spent on image processing and analysis, this could save more time in identifying patients with hyperacute stroke than some other functional MR modalities. Second, both DWI and FLAIR techniques are contrast-free image modalities, which make them safer and with fewer contrast-related complications in an emergency.

There are still several limitations in our study that should be discussed. First, the ischemic model established in our study resembles the situation of a tandem occlusion in humans that will seriously impair collateral flow and cause more severe perfusion impairment. This mechanism may be only suitable for part of the stroke event. Second, we acquired the images at the interval of 1 hour, and the magic threshold of 4.5 hours for clinical thrombolysis was excluded from acquisition. Some other MR imaging sequences such as PWI, TOF, and SWI were also acquired in 1 session (the acquisition time was nearly 35 minutes in 1 instance), and we were concerned about the quality of the DWI and FLAIR images influenced by the contrast; therefore, we believe that the time point of 4.5 hours should be included in future studies. More animal studies are needed to confirm the reproducibility of our study.

\section{CONCLUSIONS}

From our study, we found that there was significant correlation between the rFLAIR and the onset time of acute stroke. The rFLAIR might be helpful to predict the onset time of ischemic events. Meanwhile, a new parameter, the degree of DWI-FLAIR mismatch proposed in our study, could be a better indicator for identifying hyperacute ischemic stroke than was the previous visual DWI-FLAIR mismatch.

\section{ACKNOWLEDGMENTS}

We thank Zhan Zhang and Jun-cheng Dai (Nanjing Medical University, Nanjing, China) for their help in statistics and Zhan-long $\mathrm{Ma}$ (Nanjing Medical University, Nanjing, China) for discussion.

\section{REFERENCES}

1. National Institute of Neurological Disorders and Stroke rt-PA Stroke Study Group. Tissue plasminogen activator for acute ischemic stroke. N Engl J Med 1995;24:1581-87

2. Wahlgren N, Ahmed N, Davalos A, et al. Thrombolysis with alte- plase 3-4.5 $\mathrm{h}$ after acute ischemic stroke (SITS-ISTR): an observational study. Lancet 2008;372:1303-09

3. Barreto AD, Martin-Schild S, Hallevi H, et al. Thrombolytic therapy for patients who wake-up with stroke. Stroke 2009;3:827-32

4. Lee KY, Latour LL, Luby M, et al. Distal hyperintense vessels on FLAIR: an MRI marker for collateral circulation in acute stroke? Neurology 2009;72:1134-39

5. Gauvrit JY, Leclerc X, Girot M, et al. Fluid-attenuated inversion recovery (FLAIR) sequences for the assessment of acute stroke: inter observer and inter technique reproducibility. J Neurol 2006;253: 631-35

6. Aoki J, Kimura K, Iguchi Y, et al. FLAIR can estimate the onset time in acute ischemic stroke patients. J Neurol Sci 2010;293:39-44

7. Thomalla G, Rossbach P, Rosenkranz M, et al. Negative fluid-attenuated inversion recovery imaging identifies acute ischemic stroke at 3 hours or less. Ann Neurol 2009;65:724-32

8. Thomalla G, Cheng B, Ebinger M, et al. DWI-FLAIR mismatch for the identification of patients with acute ischemic stroke within $4.5 \mathrm{~h}$ of symptom onset (PRE-FLAIR): a multicenter observational study. Lancet Neurol 2011;10:978-86

9. Lu SS, Liu S, Zu QQ, et al. In vivo MR imaging of intraarterially delivered magnetically labeled mesenchymal stem cells in a canine stroke model. PLoS ONE 2013;8:e54963

10. Liu S, Hu WX, Zu QQ, et al. A novel embolic stroke model resembling lacunar infarction following proximal middle cerebral artery occlusion in beagle dogs. J Neurosci Methods 2012;209:90-96

11. Ebinger M, Galinovic I, Rozanski M, et al. Fluid-attenuated inversion recovery evolution within $\mathbf{1 2}$ hours from stroke onset: a reliable tissue clock? Stroke 2010;41:250-55

12. Cheng B, Brinkmann M, Forkert ND, et al. Quantitative measurements of relative fluid-attenuated inversion recovery (FLAIR) signal intensities in acute stroke for the prediction of time from symptom onset. J Cereb Blood Flow Metab 2013;33:76-84

13. Petkova $M$, Rodrigo $S$, Lamy $C$, et al. MR imaging helps predict time from symptom onset in patients with acute stroke: implications for patients with unknown onset time. Radiology 2010;257:782-92

14. Moonis M. Intraarterial thrombolysis within the first three hours after acute ischemic stroke in selected patients. Stroke 2009;40: 2611-12

15. Meyers PM, Schumacher HC, Higashida RT, et al. Indications for the performance of intracranial endovascular neurointerventional procedures: a scientific statement from the American Heart Association Council on Cardiovascular Radiology and Intervention, Stroke Council, Council on Cardiovascular Surgery and Anesthesia, Interdisciplinary Council on Peripheral Vascular Disease, and Interdisciplinary Council on Quality of Care and Outcomes Research. Circulation 2009;119:2235-49

16. Hacke W, Kaste M, Bluhmki E, et al. Thrombolysis with alteplase 3 to 4.5 hours after acute ischemic stroke. N Engl J Med 2008;13: 1317-29 